\title{
Resveratrol prevents inflammation and oxidative stress response in LPS-induced human gingival fibroblasts by targeting the PI3K/AKT and $\mathrm{Wnt} / \beta$-catenin signaling pathways
}

\author{
Lihua $\mathrm{Li}^{1}$, Junxiong $\mathrm{Li}^{1}$, Yujiao Wang ${ }^{1}$, Xin $\mathrm{Liu}^{2}$, Siyu $\mathrm{Li}^{1}$, Yan $\mathrm{Wu}^{1}$, Wanrong Tang ${ }^{1}$ and $\mathrm{Ya} \mathrm{Qiu}^{3}$ \\ ${ }^{I}$ North Sichuan Medical College, Department of Dentistry, Nanchong, Sichuan, P.R. China. \\ ${ }^{2}$ University of Chinese Academy of Sciences, Chongqing Savaid Stomatology Hospital, Department of \\ General Dentistry, Chongqing, P.R. China. \\ ${ }^{3}$ Affiliated Hospital of North Sichuan Medical College, Nanchong, Sichuan, P.R. China.
}

\begin{abstract}
This study aimed to elucidate the anti-inflammatory and antioxidant properties of resveratrol (RSV) in human gingival fibroblasts (HGFs) following stimulation by $P$. gingivalis lipopolysaccharide (LPS). The levels of the inflammatory cytokines IL-1 $\beta$, IL-6, IL-8 and TNF $\alpha$, the activity of the antioxidant enzymes SOD and GSH-Px, and the levels of MDA, were evaluated by ELISA. It was observed that the expression of IL-1 $\beta$, IL-6, IL-8 and TNF $\alpha$ in LPS-induced HGFs was significantly downregulated by RSV in a dose-dependent manner. RSV also partly increased oxidative stress (OS)-related factors, including SOD and GSH-Px, which was accompanied by a decrease in MDA production, although the results were not statistically significant. Additionally, RSV-induced deactivation of the PI3K/AKT and Wnt/ $\beta$ catenin pathways in LPS-induced HGFs was observed by western blot analysis. Subsequently, it was demonstrated treatment with PI3K/AKT pathway inhibitor (LY294002) or Wnt/ $\beta$-catenin pathway inhibitor (Dickkopf-1, DKK-1) could further enhance the anti-inflammatory and antioxidant effects of RSV by downregulating the expression of IL-1 $1 \beta$, IL-6, IL-8 and TNF $\alpha$, and the production of MDA, and increasing the activity of SOD and GSH-Px in LPS-induced HGFs. These results suggested RSV attenuated the inflammation and OS injury of $P$. gingivalis LPS-treated HGFS by deactivating the PI3K/AKT and Wnt/ $\beta$-catenin signaling pathways.
\end{abstract}

Keywords: Resveratrol, PI3K/AKT pathway, Wnt/ $\beta$-catenin pathway, chronic periodontitis.

Received: October 10, 2020; Accepted: April 22, 2021.

\section{Introduction}

Chronic periodontitis (CP), a destructive oral disease, is currently the main reason for tooth loss in adults (Slots, 2017). CP is usually characterized by chronic inflammation associated with pathogenic bacteria, such as Porphyromonas gingivalis ( $P$. gingivalis) in subgingival plaques, resulting in soft tissue destruction, alveolar bone resorption and, eventually, tooth loss (Rovai et al., 2016). P. gingivalis can stimulate host immune response via lipopolysaccharide (LPS) and the subsequent production of inflammatory cytokines (Zenobia and Hajishengallis, 2015). In particular, the proinflammatory cytokines IL-1 $\beta$, IL- 6 , IL- 8 and TNF $\alpha$ have been demonstrated to be the most important factors involved in periodontal tissue destruction (Noh et al., 2013; Zheng et al., 2017).

Human gingival fibroblasts (HGFs), which are mainly present in the gingival connective tissue, play an important role in the formation, regeneration, function implementation and repair of periodontal tissues (Bartold et al., 2000). HGFs not only possess an active self-renewal ability, but also synthesize and degrade extracellular matrix components, such as collagen, elastic fibers and glycoproteins (Hoang

Send correspondence to Ya Quu. Affiliated Hospital of North Sichuan Medical College, 63, Wenhua Road, Shunqing District, Nanchong, Sichuan 637000, P.R. China. E-mail: qiuyadnagy@163.com. et al., 1997). Of note, HGFs are regulated by immune factors secreted by immune cells. At the same time, HGFs also produce cytokines that finally participate in local inflammation (Naruishi and Nakata, 2018; Shang et al., 2018). Furthermore, oxidative stress (OS) occurs when excessive reactive oxygen species (ROS) accumulate and exceed the compensatory antioxidant capacity of the organism (Hernández-Ríos et al., 2017). OS leads to destruction of the periodontium, in a direct way (by damaging the biomolecules) or indirect ways [by affecting the production of pro- and antioxidant factors and enzymes, such as superoxide dismutase (SOD), glutathione peroxidase (GSH-Px) and malondialdehyde (MDA)]. Thus, the suppression of inflammation and OS play a key role in the treatment of CP.

Resveratrol (RSV; 3,4',5-trihydroxystilbene) is a natural polyphenol plant antitoxin, which has a variety of biological activities, including antioxidant, anticancer and anti-inflammatory properties, among others (Yousef et al., 2017; Yang et al., 2018; Sánchez-Melgar et al., 2019). It has been reported that RSV improved experimental periodontitis in diabetic mice and inhibited LPS-induced inflammation in gingival epithelial cells (GECs) by negatively regulating TLR4 signaling (Zhen et al., 2015). However, the function and mechanism of RSV on LPS-stimulated HGFs in vitro remain unclear. More importantly, accumulating evidence suggests that the PI3K/AKT (Lei and $\mathrm{Su}, 2019$ ) and Wnt/ $\beta$-catenin 
signaling pathways (Liu et al., 2018b) are critical mediators in the regulation of cell oxidative stress and are related to the development of CP and other chronic inflammatory diseases. Thus, the present study was undertaken to evaluate the antiinflammatory and antioxidant effects of RSV in LPS-stimulated HGFs and elucidate the role of the PI3K/AKT and Wnt/ $\beta$ catenin signaling pathways in the effect of RSV.

\section{Material and Methods}

\section{Tissue collection and cell culture}

Healthy gingival tissues were collected from three systemically healthy donors (12-16 years old) following the extraction of the canine due to orthodontics. Informed written consent was obtained from each subject and their legal guardian. The study protocol was approved by the Ethics Committee of the North Sichuan Medical College. The isolated gingival tissues were stored in Dulbecco's modified Eagle's medium (DMEM, PM150220, Procell) with $5 \%$ penicillin/streptomycin (Merck KGaA, St. Louis, MO, USA) in an ice bath. Then, the tissues were incubated with DMEM containing $0.05 \%$ collagenase I (Sigma-Aldrich, St. Louis, MO, USA) and were digested with $4 \mathrm{mg} / \mathrm{mL}$ dispase II (Sigma-Aldrich, St. Louis, MO, USA) for $30 \mathrm{~min}$ at $37^{\circ} \mathrm{C}$. After termination of the digestion, the single-cell suspension was seeded into $25 \mathrm{~cm}^{2}$ air-permeable flasks and cultured in DMEM supplemented with 20\% fetal bovine serum (FBS, cat. no. E600001-0100, Sangon Biotech, Shanghai, China) and $1 \%$ penicillin/streptomycin in a humidified incubator with $5 \%$ $\mathrm{CO}_{2}$ and $37^{\circ} \mathrm{C}$. The culture medium was changed once every 3 days and cells were passaged with $0.25 \%$ Trypsin-EDTA (Beijing Solarbio Science \& Technology Co., Ltd., Beijing, China) solution until the formation of an $80-90 \%$ confluent cell monolayer. The passaged HGFs were then cultured in $10 \%$ FBS DMEM without antibiotics. HGFs were used from passage 3 to 5 .

\section{Cell treatment}

HGFs plated in 6-well plates were incubated to approximately $80 \%$ confluence. $P$. gingivalis LPS $(1 \mu \mathrm{g} / \mathrm{mL})$ was added into the DMEM without FBS for $24 \mathrm{~h}$. Untreated cells were used as a control. For screening optimum concentration, HGFs were treated with RSV (Sigma-Aldrich, St. Louis, MO, USA) for $24 \mathrm{~h}$. In addition, RSV co-transfected HGFs were treated with PI3K/AKT pathway inhibitor (LY294002) or Wnt/ $\beta$-catenin pathway inhibitor (Dickkopf-1, DKK-1) for 24 $\mathrm{h}$, followed by LPS stimulation for $30 \mathrm{~min}$. The experiment was divided into 5 groups as follows: Control, LPS, LPS+RSV, LPS+RSV+LY294002 and LPS+RSV+DKK-1 groups. The selection of RSV concentration gradient was based on previous studies (Kang et al., 2012; Zhang et al., 2018).

\section{Cell viability assay}

The viability of HGFs was monitored using a Cell Counting Kit-8 (CCK-8, BS350B, Biosharp) according to the manufacturer's instructions. Briefly, HGFs were plated at a density of $5 \times 10^{3}$ cells/well in a 96-well plate and treated with 20,40 and $80 \mu \mathrm{mol} / \mathrm{L} \mathrm{RSV}$ for $24 \mathrm{~h}$. The culture medium of each well was immediately replaced with $100 \mu \mathrm{L}$ of $10 \%$
CCK-8 reagent-DMEM and the cells were incubated for another $2.5 \mathrm{~h}$ at $37^{\circ} \mathrm{C}$ in $\mathrm{CO}_{2}$ incubator. The cell viability was measured using an enzyme linked immune monitor (Thermo Fisher Scientific, Inc., USA) at $450 \mathrm{~nm}$. The experiments were repeated three times.

\section{Western blot analysis}

HGFs were plated in a 6-well plate at a density of $1 \times 10^{6}$ cells/well. Then, the cells were treated with RSV or/and LY294002 or DKK-1 for $24 \mathrm{~h}$, followed by LPS stimulation for $30 \mathrm{~min}$. Whole-cell lysates were washed three times in phosphate-buffered saline (PBS) and lysed in RIPA buffer (P0013C, Beyotime Institute of Biotechnology, Shanghai, China) supplemented with $1 \%$ phenylmethylsulfonyl fluoride (Beyotime Institute of Biotechnology, Shanghai, China) to obtain the proteins. A bicinchoninic acid (BCA) assay kit (CWBIO, Beijing, China) was used to determine the protein concentration. An equal amount of protein $(40 \mu \mathrm{g})$ was loaded in each lane and electrophoresed together with molecular weight standards (Bio-Rad Laboratories, Inc., Hercules, CA, USA) in separate lanes on a $10 \%$ SDS polyacrylamide gel electrophoresis. Subsequently, the proteins were transferred onto polyvinylidene fluoride (PVDF) membranes (Amersham, Piscataway, NJ, USA) at $300 \mathrm{~mA}$ for $110 \mathrm{~min}$. The membranes were blocked with $10 \%$ non-fat dry milk for $1 \mathrm{~h}$ and incubated with corresponding protein antibodies or a rabbit anti- $\beta$ actin monoclonal antibody. Subsequently, the membranes were incubated with a HRP goat anti-rabbit IgG (ab6721, Abcam). The net optical density was analyzed with a gel image processing system (Image-pro Plus 6.0, Media Cybernetics, Sliver Spring, MD). The primary antibodies used were as follows: PI3K (1/1000, cat. no. ab32089, Abcam), AKT (1/10000, cat. no. ab179463, Abcam), p-PI3K (1/500, cat. no. ab182651, Abcam), p-AKT (1/5000, cat. no. ab81283, Abcam), Wnt5a (1/100, cat. no. ab229200, Abcam), $\beta$-catenin $(1 / 1000$, cat. no. ab16051, Abcam) and $\beta$-actin $(1 / 1000$, cat. no. AC026, Abclonal). Subsequently, the protein bands were visualized using the ECL system (KF001, Affinity Biosciences) and $\beta$-actin was used as a loading control. The experiments were repeated five times.

\section{Enzyme-linked immunosorbent assay (ELISA)}

The contents of IL-1 $\beta$, IL- 6 , IL- 8 , TNF $\alpha$, SOD, MDA and GSH-Px in culture supernatants were measured with ELISA kits (BioLegend, San Diego, CA, USA) according to the manufacturer's instructions. Briefly, HGFs were seeded in a 24-well plate at a density of $2 \times 10^{5} /$ well. The cells were treated with RSV and/or LY294002 or DKK-1 for $24 \mathrm{~h}$, followed by subsequent stimulation for LPS for $30 \mathrm{~min}$. Then, the plate was washed 5 times with PBS, and $50 \mu \mathrm{L}$ biotinylated antibody working solution was added to each well and kept at $37^{\circ} \mathrm{C}$ for $20 \mathrm{~min}$. After washing as aforementioned, $100 \mu \mathrm{L}$ enzyme conjugate working solution was added into each well and kept at $37^{\circ} \mathrm{C}$ for $10 \mathrm{~min}$. Then, $100 \mu \mathrm{L}$ TMB solution was added into each well and kept at $37^{\circ} \mathrm{C}$ for $15 \mathrm{~min}$ in the dark prior to the addition of $100 \mu \mathrm{L}$ stopping solution into each well. The optical absorbance values at 450 and $570 \mathrm{~nm}$ wavelength were measured using an enzyme linked immune monitor (Thermo Fisher Scientific, Inc., USA). The experiments were repeated five times. 


\section{Statistical analysis}

The data are presented as means \pm standard deviation (SD). Statistical analysis was performed with the SPSS software (version 20.0, IBM Corp., Chicago, IL, USA). Statistical significance was determined using analysis of variance (one-ANOVA). Differences were considered significant at $P<0.05$. All error bars shown represent the calculated SD across triplicate experiments.

\section{Ethics approval}

The study protocol was approved by the Ethics Committee of the North Sichuan Medical College.

\section{Results}

\section{Effect of RSV on the proliferation of HGFs induced by LPS}

Cell viability was detected using the CCK- 8 assay. Data revealed that exposure of HGFs to RSV at 20, 40 and 80 $\mu \mathrm{mol} / \mathrm{L}$ was not associated with significant cytotoxic effects (Figure 1). These results demonstrated that the effects of RSV on LPS-induced HGFs in the present study are not attributed to non-specific cytotoxicity.

\section{RSV inhibits inflammation and OS in LPS-stimulated HGFs}

Next, the anti-inflammatory effects of RSV on LPSstimulated HGFs were investigated. As presented in Figure 2A-D, LPS stimulation led to increased levels of IL-1 $\beta$, IL-6, IL- 8 and TNF $\alpha$, whereas RSV dose-dependently suppressed the production of IL-1 $\beta$, IL-6, IL- 8 and TNF $\alpha$ in LPS-induced HGFs (Figure 2A-D). The effect of $80 \mu \mathrm{mol} / \mathrm{L} \mathrm{RSV}$ treatment was statistically significant (Figure 2A-D). In addition, ELISA results revealed that LPS stimulation significantly upregulated the content of MDA but downregulated the content of SOD and GSH-Px, which was partly reversed by RSV in a dosedependent manner (Figure 2E-G).

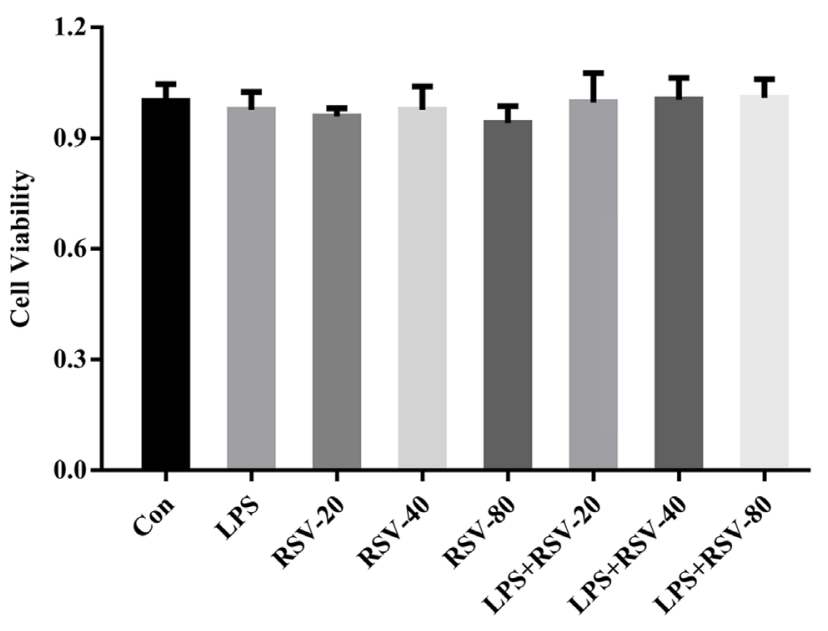

Figure 1 - The effect of RSV on the proliferation of HGFs induced by LPS. Cells were cultured with RSV at different final concentration of $20 \mu \mathrm{mol} / \mathrm{L}$ (RSV-20), $40 \mu \mathrm{mol} / \mathrm{L}$ (RSV-40), and $80 \mu \mathrm{mol} / \mathrm{L}$ (RSV-80), respectively, with and without LPS ( $1 \mu \mathrm{g} / \mathrm{mL})$ for $24 \mathrm{~h}$. Cell viability was measured by Cell Counting Kit- 8 assay. Error bars represent the SD. Statistical significance was determined with one-way ANOVA. RSV, resveratrol; HGF, human gingival fibroblast; LPS, lipopolysaccharide.

\section{RSV suppresses LPS-induced activation of the PI3K/ AKT and $W n t / \beta$-catenin signaling pathways}

To elucidate the mechanism underlying the inhibitory effects of RSV, we investigated the activation of the PI3K/ AKT and Wnt/ $\beta$-catenin signaling pathways, which play important roles in LPS-induced inflammation and OS-related cytokine production. Western blot analysis indicated that LPS significantly promoted the expression of phosphorylated (p-) PI3K, p-AKT, Wnt5a and $\beta$-catenin in HGFs (Figure 3B, $\mathrm{D}-\mathrm{G})$. As expected, RSV pretreatment $(80 \mu \mathrm{mol} / \mathrm{L})$ markedly decreased the phosphorylation levels of PI3K and AKT, as well as Wnt5a and $\beta$-catenin expression levels, in the presence of LPS (Figure 3B, D-G). However, the expression of PI3K and AKT was not changed in all treatment groups (Figure 3A, C, G).

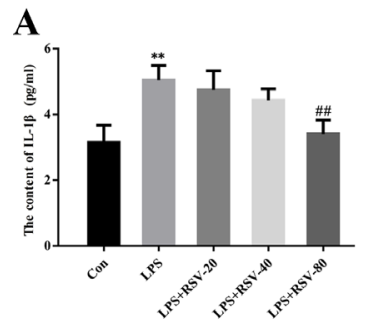

E

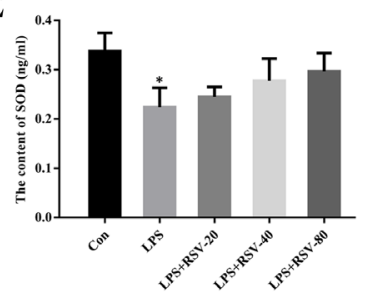

B

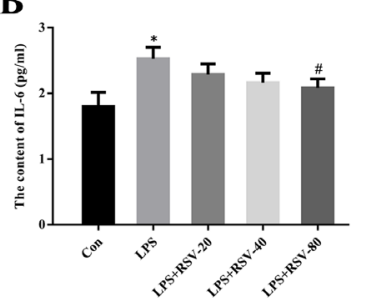

C

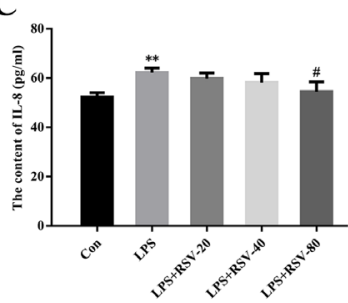

D

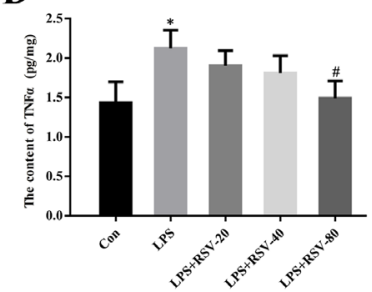

F

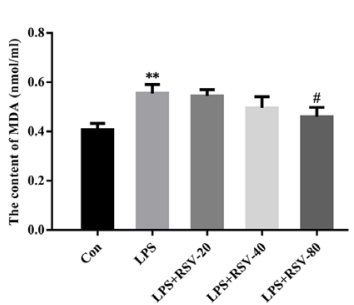

G

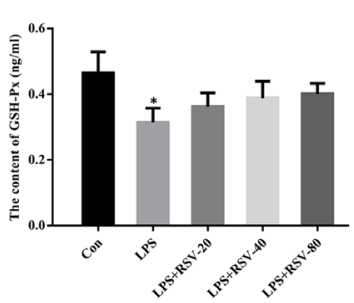

Figure 2 - RSV inhibited inflammation and OS in LPS-stimulated HGFs. HGFs were pretreated with 20 (RSV-20), 40 (RSV-40), and $80 \mu \mathrm{mol} / \mathrm{L}$ (RSV80) RSV for $24 \mathrm{~h}$, followed by LPS $(1 \mu \mathrm{g} / \mathrm{mL})$ stimulation for $24 \mathrm{~h}$. Culture supernatants were collected to evaluate the protein levels of IL-1 $\beta$ (A), IL-6 (B), IL-8 (C), TNF $\alpha$ (D), SOD (E), MDA (F) and GSH-Px (G) by ELISA. Error bars represent SD. Statistical significance was determined with one-way ANOVA. ${ }^{*} P<0.05$ (vs Control), ${ }^{* *} P<0.01$ (vs Control), ${ }^{\#} P<0.05$ (vs LPS), ${ }^{\#} P<0.01$ (vs LPS). RSV, resveratrol; HGF, human gingival fibroblast; LPS, lipopolysaccharide; OS, oxidative stress; SOD, superoxide dismutase; MDA, malondialdehyde; GSH-Px, glutathione peroxidase. 
$\mathrm{PI} 3 \mathrm{~K} / \mathrm{AKT}$ and $\mathrm{Wnt} / \beta$-catenin pathways mediate the regulatory effects of RSV on inflammation and OS in LPS-stimulated HGFs

To assess whether the PI3K/AKT and $\mathrm{Wnt} / \beta$-catenin pathways mediated pro-inflammatory effects and OS cytokine generation in HGFs, HGFs were treated with PI3K/AKT pathway inhibitor (LY294002) or Wnt/ $\beta$-catenin pathway inhibitor (DKK-1), and the cells were subsequently treated with LPS and/or RSV. Western blot analysis revealed that LY294002 decreased the phosphorylation levels of PI3K and AKT in LPS- and RSV-treated HGFs (4A, B, E). In addition, DKK-1 decreased Wnt5a and $\beta$-catenin expression levels in LPS and RSV-treated HGFs (Figure 4C-E). The ELISA results revealed that $80 \mu \mathrm{mol} / \mathrm{L} \mathrm{RSV}$ induced downregulation of IL$1 \beta$, IL-6, IL- 8 and TNF $\alpha$ levels, which were partly increased by LY294002 or DKK-1 (Figure 4F-I). Furthermore, SOD and GSH-Px were upregulated and MDA was downregulated when HGFs were treated with LPS and RSV (Figure 4J-L). Inhibition of the PI3K/AKT or $\mathrm{Wnt} / \beta$-catenin pathways in the presence of LPS and RSV further increased the levels of these cytokines (Figure 4J-L). These findings suggested that RSV inhibited inflammation and OS in LPS-stimulated HGFs partly through inactivation of the PI3K/AKT and $\mathrm{Wnt} / \beta$-catenin signaling pathways.

\section{Discussion}

Clinical periodontal treatments aimed at removing the adherent plaque bacteria by scaling, curettage and root planing are occasionally supplemented with antibiotics to control the formation of oral microorganisms. Related literature has shown that the active ingredients of natural medicines have beneficial effects on the treatment of CP due to their low toxicity, high efficiency, and the inhibition of bacterial resistance and infection (Li X et al., 2018). In the present study, the therapeutic effects of RSV on LPS-stimulated HGF inflammation were determined. The expression of the proinflammatory cytokines IL-1 $\beta$, IL-6, IL- 8 and TNF $\alpha$ were examined. LPS-stimulated HGFs exhibited higher pro-inflammatory cytokine levels, and RSV treatment significantly reduced the secretion of proinflammatory cytokines. To further verify the beneficial effect of RSV on OS injury of LPS-stimulated HGFs, the expression of antioxidant factors, including SOD, GSH-Px and MDA, was measured. It was observed that the LPS-induced decrease in SOD and GSH-Px and increase in MDA levels were partly alleviated by RSV treatment. Moreover, it was revealed that these effects of RSV were exerted through inactivation of the $\mathrm{PI} 3 \mathrm{~K} / \mathrm{AKT}$ and $\mathrm{Wnt} / \beta$-catenin signaling pathways.

$\mathrm{RSV}$ is a non-flavonoid polyphenol compound that contains a stilbene structure and is a natural non-steroidal antioxidant. RSV can be extracted from grape skin, berries and peanuts (Bhat and Pezzuto, 2002; Pervaiz, 2003; Pangeni et al., 2014). RSV has a variety of biological activities and pharmacological effects, and its anti-inflammatory and antioxidant effects have been widely studied. A previous study demonstrated that RSV inhibited LPS-induced endothelial adhesion molecule expression and vascular inflammation by inhibiting NF- $\mathrm{BB}$ activation in leukocytes (Park et al., 2009). The combination of RSV and silymarin was reported to significantly enhance the viability of fibroblasts, and RSV was able to inhibit LPS-induced IL-6 and IL-8 expression (Shahidi et al., 2017). RSV can attenuate the growth and virulence factor expression of Staphylococcus aureus, Staphylococcus epidermidis, Mycobacterium smegmatis and P. gingivalis (Sakagami et al., 2007; Kugaji et al., 2019). Thus, RSV has been considered for use in the treatment of CP. A number of signal transduction pathways and cytokines have been found to be involved in the effects of RSV on $P$. gingivalis-induced inflammation in HGFs and ligature-
A

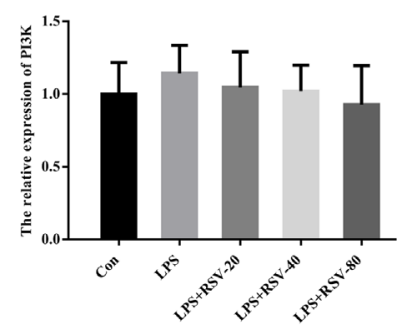

D

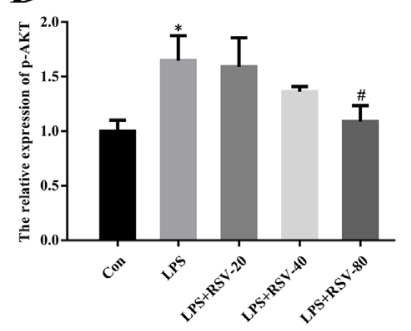

B

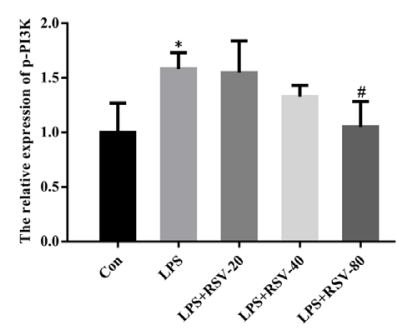

E

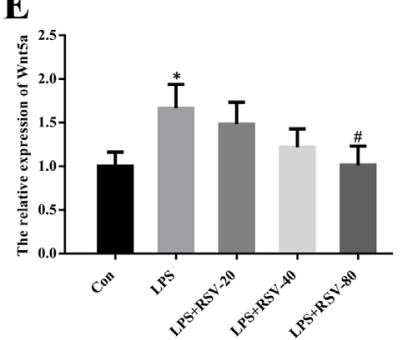

C

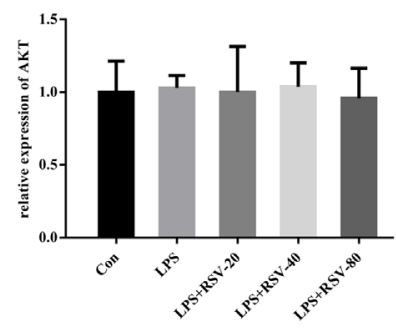

F

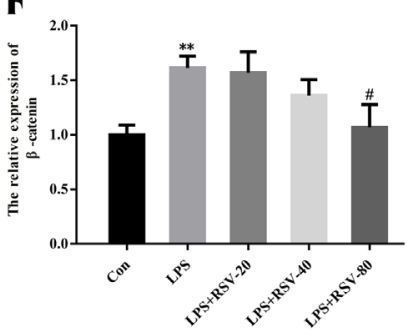

G
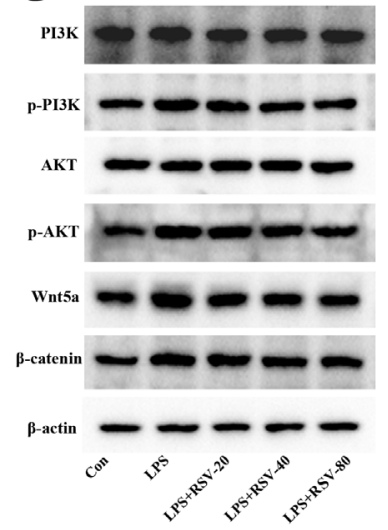

Figure 3 - RSV suppressed LPS-induced activation of the PI3K/AKT and Wnt/ $\beta$-catenin signaling pathways. HGFs were pretreated with 20 (RSV-20), 40 (RSV-40), and $80 \mu \mathrm{mol} / \mathrm{L}$ (RSV-80) RSV for $24 \mathrm{~h}$, followed by LPS ( $1 \mu \mathrm{g} / \mathrm{mL})$ stimulation for $24 \mathrm{~h}$. The expression of PI3K, p-PI3K, AKT, p-AKT, Wnt5a and $\beta$-catenin was determined by western blot analysis. $\beta$-actin served as the loading control. Error bars represent SD. Statistical significance was determined with one-way ANOVA. " $P<0.05$ (vs Control), ${ }^{* *} P<0.01$ (vs Control), ${ }^{\#} P<0.05$ (vs LPS). RSV, resveratrol; HGF, human gingival fibroblast; LPS, lipopolysaccharide. 
A

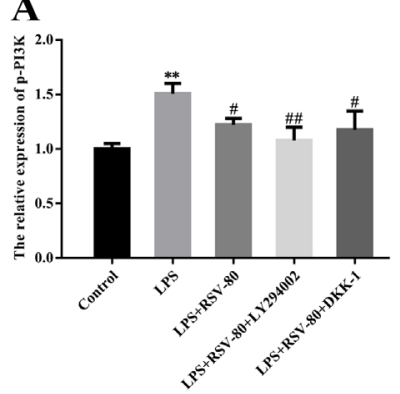

E

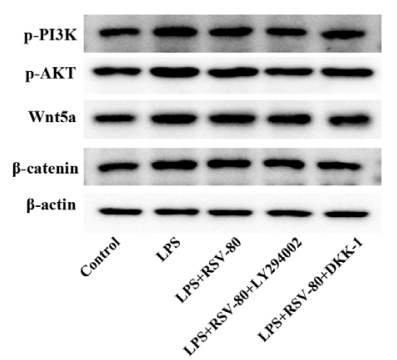

I

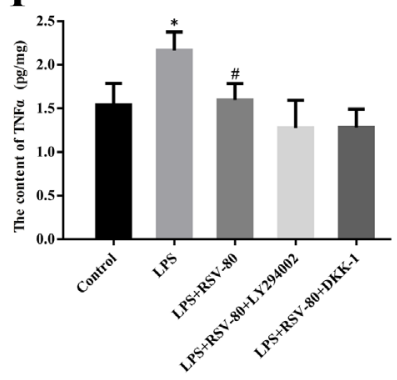

B
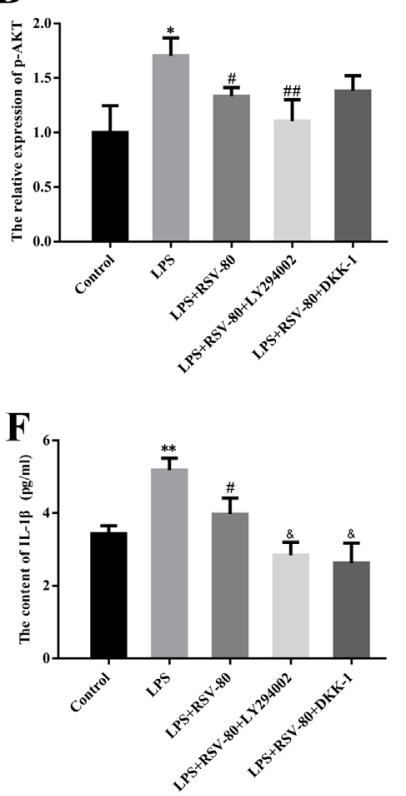

.I

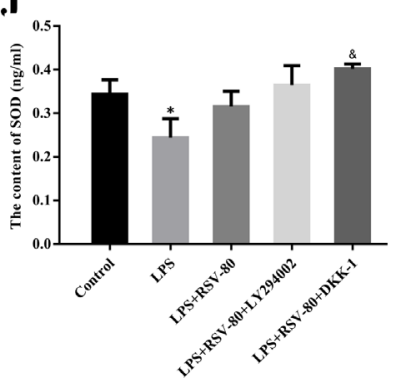

C

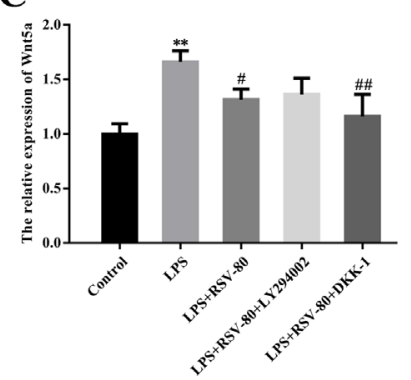

G

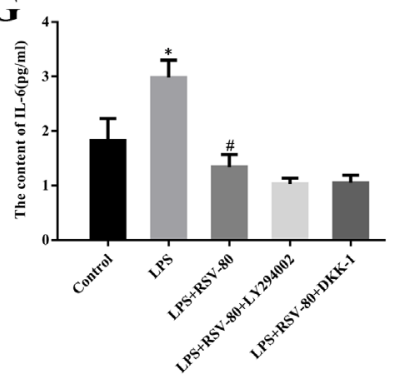

K

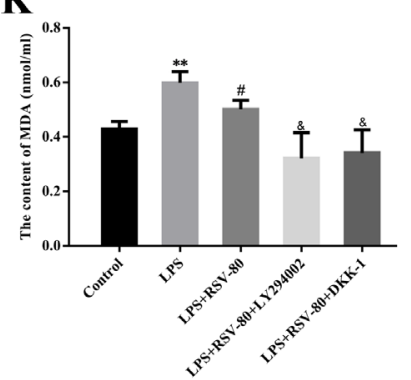

D

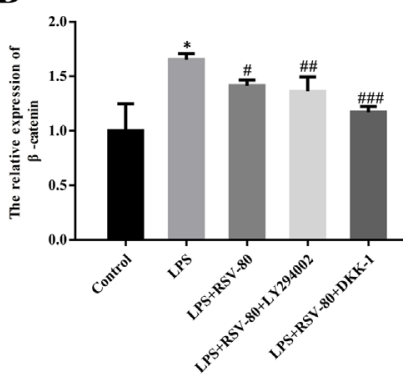

H

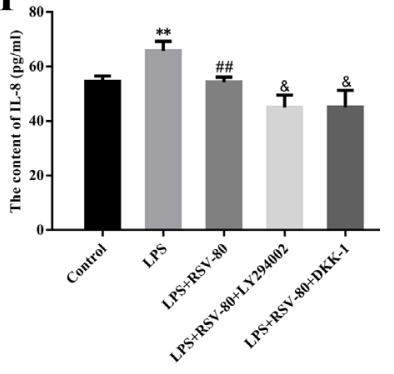

L

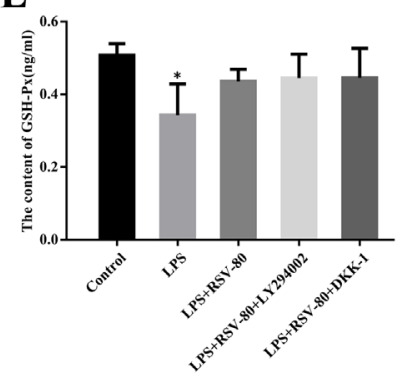

Figure 4 - PI3K/AKT and Wnt/ $\beta$-catenin pathways mediated the regulatory effects of RSV on inflammation and OS of LPS-stimulated HGFs. HGFs were treated with PI3K/AKT pathway inhibitor (LY294002) or Wnt/ $\beta$-catenin pathway inhibitor (DKK-1), followed by treatment with LPS and/or RSV. (A-E) The expression of p-PI3K, p-AKT, Wnt5a and $\beta$-catenin was determined by western blot analysis. $\beta$-actin served as the loading control. Culture supernatants were collected to evaluate the protein levels of IL-1 $\beta$ (F), IL-6 (G), IL-8 (H), TNF $\alpha$ (I), SOD (J), MDA (K) and GSH-Px (L) by ELISA. Error bars represent SD. Statistical significance was determined with one-way ANOVA. ${ }^{*} P<0.05$ (vs Control), ${ }^{* *} P<0.01$ (vs Control), ${ }^{*} P<0.05$ (vs LPS), ${ }^{\#} P<0.01$ (vs LPS), ${ }^{\# \#} P<0.001$ (vs LPS), ${ }^{\circledR} P<0.05$ (vs LPS+RSV-40). RSV, resveratrol; HGF, human gingival fibroblast; LPS, lipopolysaccharide; OS, oxidative stress; SOD, superoxide dismutase; MDA, malondialdehyde; GSH-Px, glutathione peroxidase.

induced periodontitis animal models. RSV led to alveolar bone resorption and decreased IL-17 expression in rats with experimental periodontitis (Casati et al., 2013). Similar data demonstrated that RSV effectively promoted osteogenesis mainly via SIRT1/FOXO3A signaling and UNX2 gene expression (Tseng et al., 2011). The present study proved that treatment with RSV did not significantly affect HGF viability, and a previous study reported that RSV inhibited the secretion of IL-1 $\beta$, IL- 6 , IL- 8 and TNF $\alpha$ induced by LPS in HGFs (Buranasin and Mizutani, 2018).

It is known that OS is considered to be a key factor implicated in cell injury. Excessive oxygen free radical accumulation usually causes lipid peroxidation and protein oxidative damage in the cell membrane, thereby destroying cell membrane integrity and inducing cell inflammation or apoptosis. Several studies suggested that OS mediates the proliferation, apoptosis and migration of HGFs, as well as gingival wound healing (Cheng et al., 2015; Kido et al., 2017; Buranasin and Mizutani, 2018). It was demonstrated that, when HGFs are exposed to LPS/streptococcal cell walls (SCW)/formyl-methionyl-leucyl-phenylalanine, the manganese superoxide dismutase (MnSOD) expression is upregulated (Skaleric et al., 2000). It is known that oxidant and antioxidant factors, such as SOD, GSH-Px and MDA, are crucial OS-related factors, and OS has been implicated in the development and progression of periodontitis (Tonguç et al., 2011; Özdem et al., 2017). The present study revealed elevated MDA level as well as reduced SOD and GSH-Px levels in the model group, whereas RSV partially reversed these changes in expression, indicating that RSV may alleviate oxidative stress in LPS-induced HGFs.

Mechanically, RSV could inhibit cancer cell proliferation and invasiveness via inhibition of the PI3K/AKT and Wnt/ $\beta$ catenin pathways (Tsai et al., 2013; Liu et al., 2014). Previous reports have revealed that the $\mathrm{PI} 3 \mathrm{~K} / \mathrm{AKT}$ and $\mathrm{Wnt} / \beta$-catenin pathways are critical mediators during the treatment of periodontal disease (Nakayama et al., 2015; Miranda et al., 2017). In gingival epithelial cells, $P$. gingivalis was shown to regulate $\mathrm{PI} 3 \mathrm{~K}$ and $\mathrm{AKT}$ and the phosphorylation levels of the AKT downstream proteins GSK3, mTOR and Bad, which is 
linked to cell survival and immune responses (Nakayama et al., 2015). It was recently found that LPS from $P$. gingivalis induced autophagy of HGFs via the PI3K/AKT signaling pathway (Liu et al., 2018a). In addition, plantamajoside, caffeic acid phenethyl ester and farrerol all suppressed the inflammatory response in LPS-stimulated HGFs through inhibiting the PI3K/AKT signaling pathway (Wang et al., 2016; Li L et al., 2017; Liu et al., 2019). In an experimental periodontal disease model, gliclazide treatment reduced myeloperoxidase activity, MDA, IL- $1 \beta$ and TNF- $\alpha$ levels via downregulation of PI3K and AKT (Araújo and Morais, 2019).

Emerging evidence demonstrated that DKK1, an antagonist of $\mathrm{Wnt} / \beta$-catenin signaling, suppressed bone formation and contributed to inflammation in periodontitis (Miranda et al., 2017). In addition, higher levels of Wnt3a, $\beta$-catenin and matrix metalloproteinase inducer (EMMPRIN) were observed in gingival tissues of CP. Furthermore, LPS significantly enhanced $\beta$-catenin induction in a human immortalized oral epithelial cell (HIOEC)/HGF co-culture model (Liu et al., 2018b). In addition, in periodontitis, aberrant activation of the Wnt/ $\beta$-catenin signaling pathway could inhibit the EMMPRIN/MMP-2/ 9 axis (Liu et al., 2018b). The Wnt antagonist sFRP1 significantly reduced bone loss of periapical lesions in vivo (Yang et al., 2021). In human periodontal ligament fibroblasts (hPLFs), knockdown of $\beta$-catenin or treatment with DKK1 facilitated $\mathrm{H}_{2} \mathrm{O}_{2}$-induced oxidative damage (Kook et al., 2016). Therefore, it was hypothesized that RSV suppressed the inflammation and OS injury in LPS-induced HGFs through attenuating the PI3K/ $\mathrm{AKT}$ and $\mathrm{Wnt} / \beta$-catenin signaling pathways. The data of the present study support this hypothesis, as activated PI3K/ AKT and $W n t / \beta$-catenin signaling was observed after LPS stimulation. Furthermore, RSV partly inhibited the activation of the PI3K/AKT and Wnt/ $\beta$-catenin signaling pathways. In HGFs, co-treatment with LPS and RSV, and specific inhibition of PI3K/AKT (LY294002) and Wnt/ $\beta$-catenin (DKK-1), further reduced inflammation and $\mathrm{OS}$.

\section{Conclusions}

The present study provided evidence and discussed the mechanisms of action of RSV in LPS-stimulated HGFs. RSV was shown to attenuate the inflammatory and OS response in LPS-stimulated HGFs by inhibiting the PI3K/AKT and $\mathrm{Wnt} / \beta$-catenin signaling pathways.

\section{Acknowledgements}

This study was supported by a grant from Nanchong City School Science and Technology Strategic Cooperation Project [grant number 18SXHZ0092], the Nanchong City School Science and Technology Strategic Cooperation Project [grant number 19SXHZ0078], and Sichuan Provincial Department of Education [grant number 15zb0199].

\section{Conflict of Interest}

The authors declare that they have no competing interests.

\section{Authors Contributions}

LL, JL, and YW conceived and designed the study; LL, JL, XL, SL, YW conducted the experiments; LL, YW, WT analyzed the data and confirmed the authenticity of the raw data; LL wrote the manuscript; YQ contributed to the reagents and materials. All the authors have read and approved the final version of the manuscript.

\section{References}

Araújo AA and Morais HB (2019) Gliclazide reduced oxidative stress, inflammation, and bone loss in an experimental periodontal disease model. J Appl Oral Sci 27:e20180211.

Bartold PM, Walsh LJ and Narayanan AS (2000) Molecular and cell biology of the gingiva. Periodontol 2000 24:28-55.

Bhat KP and Pezzuto JM (2002) Cancer chemopreventive activity of resveratrol. Ann New York Acad Sci 957:210-229.

Buranasin P and Mizutani K (2018) High glucose-induced oxidative stress impairs proliferation and migration of human gingival fibroblasts. PLoS One 13:e0201855.

Casati MZ, Algayer C, Cardoso da Cruz G, Ribeiro FV, Casarin RC, Pimentel SP and Cirano FR (2013) Resveratrol decreases periodontal breakdown and modulates local levels of cytokines during periodontitis in rats. J Periodontol 84:e58-64.

Cheng R, Choudhury D, Liu C, Billet S, Hu T and Bhowmick NA (2015) Gingival fibroblasts resist apoptosis in response to oxidative stress in a model of periodontal diseases. Cell Death Discov 1:15046.

Hernández-Ríos P, Pussinen PJ, Vernal R and Hernández M (2017) Oxidative stress in the local and systemic events of apical periodontitis. Front Physiol 8:869.

Hoang AM, Chen D, Oates TW, Jiang C, Harris SE and Cochran DL (1997) Development and characterization of a transformed human periodontal ligament cell line. J Periodontol 68:10541062.

Kido D, Mizutani K, Takeda K, Mikami R, Matsuura T, Iwasaki K and Izumi Y (2017) Impact of diabetes on gingival wound healing via oxidative stress. PLoS One 12:e0189601.

Kang NE, Ha AW, Kim JY and Kim WK (2012) Resveratrol inhibits the protein expression of transcription factors related adipocyte differentiation and the activity of matrix metalloproteinase in mouse fibroblast 3T3-L1 preadipocytes. Nutr Res and Pract 6:499-504.

Kook SH, Lee D, Cho ES, Heo JS, Poudel SB, Ahn YH, Hwang JW, Ji H, Kim JG and Lee JC (2016) Activation of canonical $\mathrm{Wnt} / \beta$-catenin signaling inhibits $\mathrm{H} 2 \mathrm{O} 2$-induced decreases in proliferation and differentiation of human periodontal ligament fibroblasts. Mol Cell Biochem 411:83-94.

Kugaji MS, Kumbar VM, Peram MR, Patil S, Bhat KG and Diwan PV (2019) Effect of Resveratrol on biofilm formation and virulence factor gene expression of Porphyromonas gingivalis in periodontal disease. APMIS 127:187-195.

Lei $\mathrm{S}$ and $\mathrm{Su} \mathrm{W}$ (2019) Hyperglycemia-induced oxidative stress abrogates Remifentanil preconditioning-mediated cardioprotection in diabetic rats by impairing Caveolin-3modulated PI3K/Akt and JAK2/STAT3 signaling. Oxid Med Cell Longev 2019:9836302.

Li L, Sun W, Wu T, Lu R and Shi B (2017) Caffeic acid phenethyl ester attenuates lipopolysaccharide-stimulated proinflammatory responses in human gingival fibroblasts via NF- $\kappa \mathrm{B}$ and PI3K/ Akt signaling pathway. Eur J Pharmacol 794:61-68. 
Li X, Yu C, Hu Y, Xia X, Liao Y, Zhang J, Chen H, Lu W, Zhou W and Song Z (2018) New application of Psoralen and Angelicin on periodontitis with anti-bacterial, anti-inflammatory, and osteogenesis effects. Front Cell Infect Microbiol 8:178.

Liu F, Huang X, He JJ, Song C, Peng L, Chen T and Wu BL (2019) Plantamajoside attenuates inflammatory response in LPSstimulated human gingival fibroblasts by inhibiting PI3K/AKT signaling pathway. Microb Pathog 127:208-211.

Liu J, Wang X, Zheng M and Luan Q (2018a) Lipopolysaccharide from Porphyromonas gingivalis promotes autophagy of human gingival fibroblasts through the PI3K/Akt/mTOR signaling pathway. Life Sci 211:133-139.

Liu X, Zhang Z, Pan S, Shang S and Li C (2018b) Interaction between the Wnt/ $\beta$-catenin signaling pathway and the EMMPRIN/ MMP-2, 9 route in periodontitis. J Periodontal Res 53:842-852.

Liu YZ, Wu K, Huang J, Liu Y, Wang X, Meng ZJ, Yuan SX, Wang DX, Luo JY et al. (2014) The PTEN/PI3K/Akt and Wnt/Bcatenin signaling pathways are involved in the inhibitory effect of resveratrol on human colon cancer cell proliferation. Int $\mathrm{J}$ Oncol 45:104-112.

Miranda TS, Napimoga MH, Feres M, Marins LM, Cruz DFD, Silva HDPD and Duarte PM (2017) Antagonists of Wnt $/ \beta$-catenin signaling in the periodontitis associated with type 2 diabetes and smoking. J Clin Periodontol 45:293-302.

Noh MK, Jung M, Kim SH, Lee SR, Park KH, Kim DH, Kim HH and Park YG (2013) Assessment of IL-6, IL-8 and TNF- $\alpha$ levels in the gingival tissue of patients with periodontitis. Exp Ther Med 6:847-851.

Nakayama M, Inoue T, Naito M, Nakayama K and Ohara N (2015) Attenuation of the phosphatidylinositol 3-kinase/Akt signaling pathway by Porphyromonas gingivalis gingipains RgpA, RgpB, and Kgp. J Biol Chem 290:5190-5202.

Naruishi K and Nagata T (2018) Biological effects of interleukin-6 on Gingival Fibroblasts: Cytokine regulation in periodontitis. J Cell Physiol 233:6393-6400.

Özdem M, Kırzıoğlu FY, Yılmaz HR, Vural H, Fentoğlu Ö, Uz E, Koçak A and Yiğit A (2017) Antioxidant effects of melatonin in heart tissue after induction of experimental periodontitis in rats. J Oral Sci 59:23-29.

Pervaiz S (2003) Resveratrol: from grapevines to mammalian biology. FASEB J 17:1975-1985.

Park HJ, Jeong SK, Kim SR, Bae SK, Kim WS, Jin SD, Koo TH, Jang HO, Yun I, Kim KW, et al (2009) Resveratrol inhibits Porphyromonas gingivalis lipopolysaccharide-induced endothelial adhesion molecule expression by suppressing NFkappaB activation. Arch Pharm Res 32:583-591.

Pangeni R, Sahni JK, Ali J, Sharma S and Baboota S (2014) Resveratrol: review on therapeutic potential and recent advances in drug delivery. Expert Opin Drug Deliv 11:1285-1298.

Rovai ES, Souto ML, Ganhito JA, Holzhausen M, Chambrone L and Pannuti CM (2016) Efficacy of local antimicrobials in the non-surgical treatment of patients with periodontitis and Diabetes: A systematic review. J Periodontol 87:1406-1417.

Skaleric U, Manthey CM, Mergenhagen SE, Gaspirc B and Wahl SM (2000) Superoxide release and superoxide dismutase expression by human gingival fibroblasts. Eur J Oral Sci 108:130-135.

Sakagami Y, Sawabe A, Komemushi S, All Z, Tanaka T, Iliya I and Iinuma M (2007) Antibacterial activity of stilbene oligomers against vancomycin-resistant Enterococci (VRE) and methicillin-resistant Staphylococcus aureus (MRSA) and their synergism with antibiotics. Biocontrol Sci 12:7-14.

Slots J (2017) Periodontitis: facts, fallacies and the future. Periodontology 2000 75:7-23.
Shahidi M, Vaziri F, Haerian A, Farzanegan A, Jafari S, Sharifi R and Shirazi FS (2017) Proliferative and anti-inflammatory effects of Resveratrol and Silymarin on human gingival fibroblasts: A view to the future. J Dent (Tehran) 14:203-211

Shang L, Wang T, Tong D, Kang W, Liang Q and Ge S (2018) Prolyl hydroxylases positively regulated LPS-induced inflammation in human gingival fibroblasts via TLR4/MyD88-mediated AKT/NF- $\mathrm{BB}$ and MAPK pathways. Cell Prolif 51:e12516.

Sánchez-Melgar A, Albasanz JL, Guixà-González R, Saleh N, Selent J and Martín M (2019) The antioxidant resveratrol acts as a non-selective adenosine receptor agonist. Free Radic Biol Med 135:261-273.

Tonguç M, Öztürk O, Sütçü R, Ceyhan BM, Kılınç G, Sönmez Y, Yetkin Ay Z, Sahin U, Baltacıoğlu E and Kırzıoğlu FY (2011) The impact of smoking status on antioxidant enzyme activity and malondialdehyde levels in chronic periodontitis. J Periodontol 82:1320-1328.

Tseng PC, Hou SM, Chen RJ, Peng HW, Hsieh CF, Kuo ML and Yen ML (2011) Resveratrol promotes osteogenesis of human mesenchymal stem cells by upregulating RUNX2 gene expression via the SIRT1/FOXO3A axis. J J Bone Miner Res 26:2552-2563.

Tsai JH, Hsu LS, Lin CL, Hong HM, Pan MH, Way TD and Chen WJ (2013) 3,5,4'-Trimethoxystilbene, a natural methoxylated analog of resveratrol, inhibits breast cancer cell invasiveness by downregulation of PI3K/Akt and Wnt/ $\beta$-catenin signaling cascades and reversal of epithelial-mesenchymal transition. Toxicol Appl Pharmacol 272:746-756.

Wang Q, Zhang B and Yu JL (2016) Farrerol inhibits IL-6 and IL-8 production in LPS-stimulated human gingival fibroblasts by suppressing PI3K/AKT/NF- $\mathrm{KB}$ signaling pathway. Arch Oral Biol 62:28-32.

Yousef M, Vlachogiannis IA and Tsiani E (2017) Effects of Resveratrol against Lung Cancer: In vitro and In vivo studies. Nutrients 9:1231.

Yang F, Huang D, Xu L, Xu W, Yi X, Zhou X, Ye L, Zhang L (2021) Wnt antagonist secreted frizzled-related protein I (sFRP1) may be involved in the osteogenic differentiation of periodontal ligament cells in chronic apical periodontitis. Int Endod J. 54:768-779.

Yang L, Zhang Z, Zhuo Y, Cui L, Li C, Li D, Zhang S, Cui N, Wang $X$ and Gao H (2018) Resveratrol alleviates sepsis-induced acute lung injury by suppressing inflammation and apoptosis of alveolar macrophage cells. Am J Transl Res 10:1961-1975.

Zenobia C and Hajishengallis G (2015) Porphyromonas gingivalis virulence factors involved in subversion of leukocytes and microbial dysbiosis. Virulence 6:236-243.

Zhang Z, Liu Z, Chen J, Yi J, Cheng J, Dun W and Wei H (2018) Resveratrol induces autophagic apoptosis via the lysosomal cathepsin D pathway in human drug-resistant K562/ADM leukemia cells. Exp Ther Med 15:3012-3019.

Zhen L, Fan DS, Zhang Y, Cao XM and Wang LM (2015) Resveratrol ameliorates experimental periodontitis in diabetic mice through negative regulation of TLR4 signaling. Acta Pharmacolo Sin $36: 221-228$.

Zheng XY, Mao CY, Qiao H, Zhang X, Yu L, Wang TY and Lu EY (2017) Plumbagin suppresses chronic periodontitis in rats via down-regulation of TNF- $\alpha$, IL- $1 \beta$ and IL- 6 expression. Acta Pharmacol Sin 38:1150-1160.

Associate Editor: Carlos F. M. Menck

License information: This is an open-access article distributed under the terms of the Creative Commons Attribution License (type CC-BY), which permits unrestricted use distribution and reproduction in any medium, provided the original article is properly cited. 